\title{
The impact of emotions and predominant emotion regulation technique on driving performance
}

\author{
G. M. Hancock ${ }^{\mathrm{a}}{ }^{1}$, P. A. Hancock ${ }^{\mathrm{b}}$ and C.M. Janelle ${ }^{\mathrm{c}}$ \\ ${ }^{a}$ Performance Research Laboratory, Department of Psychology, University of Central Florida, Orlando, FL \\ ${ }^{\mathrm{b}}$ MIT ${ }^{2}$ Laboratory, Department of Psychology, University of Central Florida, Orlando, FL \\ ${ }^{\mathrm{c}}$ Performance Psychology Laboratory, Department of Applied Physiology and Kinesiology, University of \\ Florida, Gainesville, FL
}

\begin{abstract}
Emotion-provoking stimuli abound on modern roadways. Driving measures, of both longitudinal and lateral control of the vehicle, have been shown to vary based on affective influences. Research, however, has yet to address how drivers' individual techniques to mitigate emotional reactions influence driving performance. To address this issue, the present study featured a dual-task protocol involving simulated driving together with processing of emotionally-valenced images with a focus on different Predominant Emotion Regulation Techniques (PERT): one adaptive strategy (task-focused coping) and one maladaptive style (emotion-focused coping). Dependent measures included mean driving speed and number of lane excursions. Results indicated that pleasant images degraded longitudinal control to the greatest extent, while unpleasant images produced the greatest detriment in lateral control. Additionally, individuals' PERT played a major interactive role in drivers' longitudinal control leading task-focused females and emotion-focused males to adhere more closely to the speed limit; yet, it did not affect their lateral control. Results hold important potential implications for the amount or variety of training necessary for driver licensure to promote and sustain safe vehicle control.
\end{abstract}

Keywords: Vehicle Operation Efficacy, Affective States, Traffic Safety

${ }^{1}$ Corresponding author: 4000 Central Florida Boulevard, Orlando, FL 32816-1390.

Telephone: (407) 823 - 4344. Fax: (407)823 - 5862. E-mail: g.hancock@knights.ucf.edu, gh826@hhp.ufl.edu 


\section{Introduction}

Driving a vehicle is typically a routine activity. However, any drive has the potential to be intensely emotional due to the risks of injury and death. Driving while emotional is commonplace on the road and emotional drivers exhibit less control of the steering wheel [11] and more extreme use of the pedals [10], leading to an increased risk of an accident $[2,11]$. Traffic collisions, often stemming from unstable emotional states, bear costs in terms of life, limb and revenue.

Both the psychological and physical components of the driving task vary based on emotional state [9]. In his Transactional Model of Driver Stress and Fatigue, Matthews [6] suggests that environmental factors (unpredictable environmental stimuli) and personality factors (predispositions guiding the interpretation of stimuli) bias cognitive stress processes (perceptions and responses to stress). Cognitive stress processes subsequently influence subjective and objective performance. Certain individuals are theorized to perform superiorly as their cognitive stress processes are more adapted to situational demands. Adaptive versus maladaptive emotion regulation strategies were therefore compared. Task-focused coping involves channeling emotional reactivity in productive, task-related ways while emotion-focused coping leads drivers to dwell on their emotional reactivity, distracting them from performing effectively [1].

The potential for emotion regulation (ER) to impact driving performance is clear, yet emphasis is consistently placed on emotional reactivity rather than ER. How predominant ER technique influences driving performance is unknown. To address this limitation, the performance of drivers who favor different ER strategies was evaluated. The dependent measures were mean driving speed (as expressed by a percentage of the speed limit) and lane excursions [3]. Adaptive regulators were hypothesized to drive more safely than maladaptive regulators by exhibiting smaller mean speeds (i.e., adhering closer to the speed limit) and less lane excursions.

\section{Methods}

72 participants $(50 \%$ male) were recruited from a university campus. Participants were divided evenly between task-focused and emotion-focused copers. Exclusion criteria consisted of: a history of simulator sickness, a medication regimen which affected their cardiovascular system, or if participants' age exceeded 40 years old.

PERT was determined via scores on the Driver Coping Questionnaire (DCQ) [7]. The DCQ is a 35-item questionnaire which gauges how people typically react to difficult or stressful driving scenarios. While the battery has 5 distinct ER strategies/subscales, this study focused on one representative adaptive technique (i.e., task-focused coping) and one representative maladaptive style (i.e., emotion-focused coping). A fixed-base driving simulator and a mini notebook laptop (placed in roughly the same location as a GPS device) presented the protocol. The protocol involved a dual-task paradigm including simulated driving and concurrent processing of emotional stimuli. Participants were instructed to drive as safely as possible in the simulated environment (i.e., adhering as closely as possible to the $30 \mathrm{mph}$ speed limit and maintaining their lane position to the best of their ability) while monitoring the laptop screen for detour signs which would demarcate the correct route to take.

Emotions were manipulated via images of different valences (i.e., pleasant, unpleasant, neutral) which were randomly presented on the same screen as the detour signs. General affective images were selected from the International Affective Picture System [4]. Driving-related images were selected and validated by matching for the IAPS images' valence and arousal scores.

Driving data were collected via a fixed-base driving simulator and a Labview program. Mean speeds were derived from instantaneous speeds in response to affective images and expressed as a percentage of the speed limit. Lane excursions were tallied as the number of instances drivers violated the boundaries of their lane in response to the affective images.

\section{Results}

Multiple 2 (SEX: male, female) x 3 (VALENCE: pleasant, unpleasant, neutral) x 2 (PREDOMINANT EMOTION REGULATION TECHNIQUE: taskfocused coping; emotion-focused coping) mixed model ANOVAs were run on each driving measure. Separate analyses were likewise conducted for reactions to driving-related versus non driving-specific (i.e., IAPS) images. 


\section{Mean Driving Speeds}

Images' valence had a significant effect on mean driving speeds $(F=6.381, p=.002)$. As illustrated in Figure 1, pleasant images prompted significantly slower speeds when compared to unpleasant images (mean difference $=7.078 \mathrm{mph}, p=.001$ ) and neutral pictures (mean difference $=4.294, p=.034$ ).

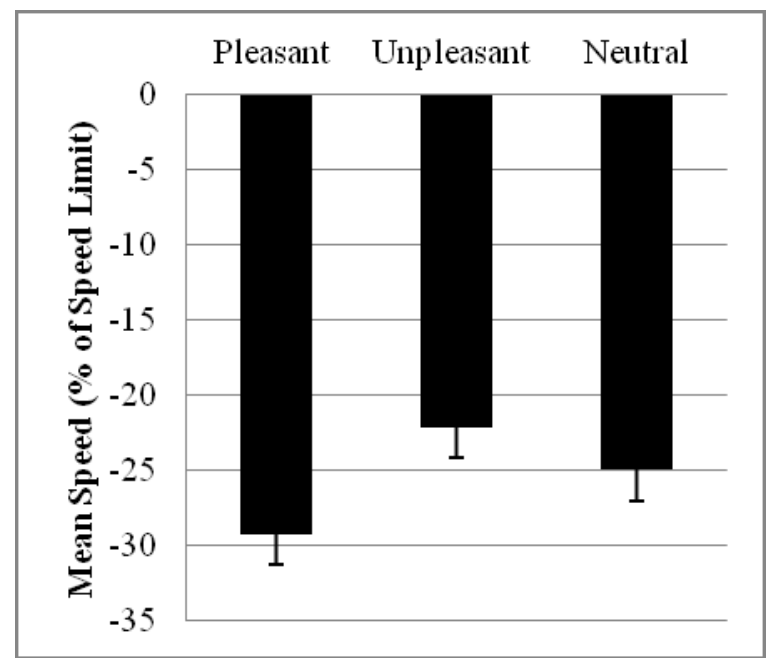

Fig. 1: Main effect for valence on mean driving speeds in response to all images. Pleasant images prompted significantly slower speeds when compared to unpleasant and neutral images. Error bars are standard errors.

Mean driving speeds in response to domain-specific images follow this same pattern, with pleasant images eliciting significantly slower speeds when compared to unpleasant (mean difference $=9.980, p=$ .001 ) and neutral scenes (mean difference $=7.343, p$ $=.040$ ). Additionally, there was a significant Sex $\mathrm{x}$ PERT interaction $(F=8.194, p=.006)$. Taskfocused females drove closer to the speed limit, while emotion-focused males adhering more closely to the speed limit (Figure 2). There were no significant effects or interactions for mean driving speeds in reaction to IAPS (non driving-specific) images.

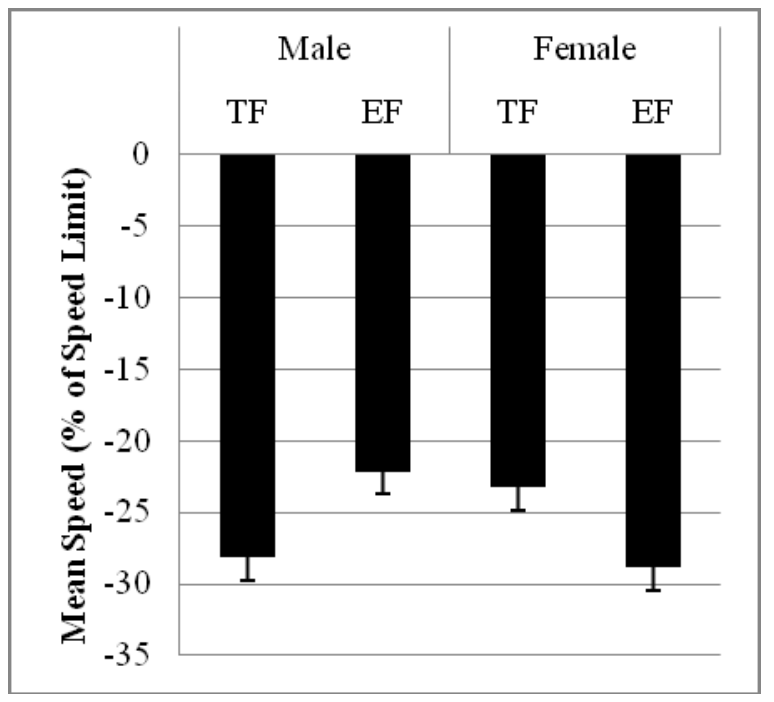

Fig. 2: Sex $x$ PERT interaction on mean driving speeds. Taskfocused (TF) females and emotion-focused (EF) males drive more safely (i.e., closer to the speed limit). Error bars are standard errors.

\section{Lane Excursions}

As presented in Figure 3, there was a main effect for valence on lane excursions for all images $(F=5.977$, $\mathrm{p}=.010)$. Unpleasant images provoked significantly more lane excursions when compared to pleasant (mean difference $=.209, p=.022)$ and neutral scenes (mean difference $=.208, p=.004)$.

Driving-specific images produced the same pattern of response: main effect for valence $(F=6.995, p=$ $.004)$ with unpleasant scenes inciting significantly more lane excursions than pleasant (mean difference $=.178, p=.003$ ) and neutral (mean difference $=$ $.117, p=.034$ ). Additionally, as depicted in Figure 4, driving-specific neutral images caused significantly more lane excursions than driving-specific images (mean difference $=.061, p=.043$ ). 


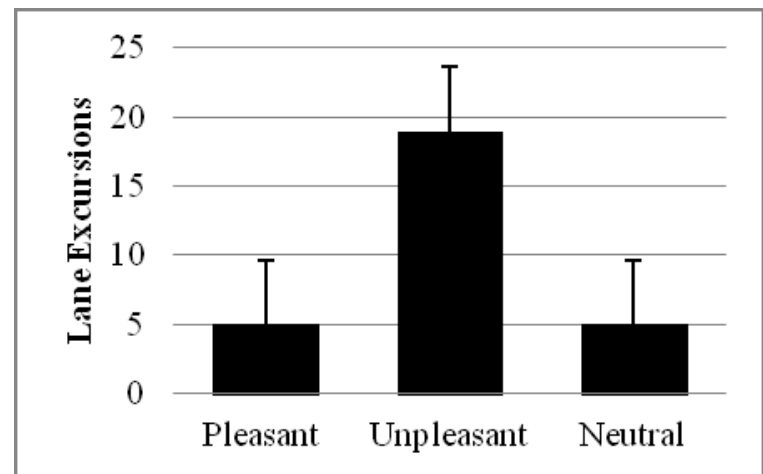

Fig. 3: Overall lane excursions by valence for all images. Error bars are standard errors.

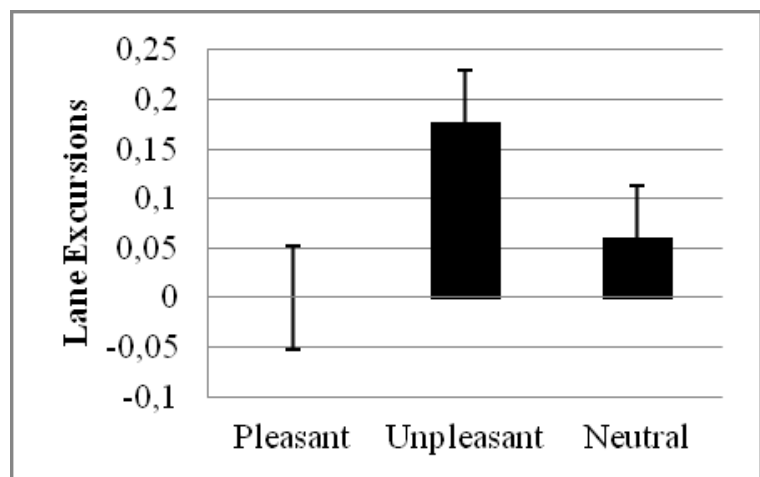

Fig. 4: Average lane excursions by valence in response to drivingrelated images. Error bars are standard errors.

As with mean driving speed results, the presentation of IAPS images did not result in any significant effects or interactions on number of lane excursions.

\section{Discussion}

Affective stimuli presented during dual-task driving cause pronounced changes in driving efficacy. Results clarify equivocal research concerning effects of pleasant emotions on driving $[8,9]$. Pleasant images compromised longitudinal control to the greatest extent in the current study by prompting slower speeds (Figure 1), which corroborates the work of Pêcher and colleagues [9].

Based on mean speeds, task-focused females and emotion-focused males drove more closely to the speed limit (Figure 1). Young males apparently establish maladaptive (though effective) ER strategies early in their development; these techniques then foster dangerous, long-term behaviors which potentially fuel males' higher rates of traffic-related injuries and citations [5].
Contrary to Pêcher and associates' findings [9], unpleasant images were found to provoke the most lane excursions (Figure 3). Such elevated numbers of lane excursions after exposure to unpleasant images may be due to their depiction of driving's aversive consequences, especially as this effect is seen only in response to driving-related images. Results regarding the longitudinal and lateral control of the vehicle may have implications for the amount or variety of training necessary for licensure. Additional training to reinforce the adoption and use of adaptive versus maladaptive strategies may help inexperienced drivers establish safe, life-long driving habits.

\section{References}

[1] Desmond, P.A., \& Matthews, G. (2009). Individual differences in stress and fatigue in two field studies of driving. Transportation Research Part F: Traffic Psychology and Behaviour, 12, 265-276.

[2] Groeger, J.A. (1997). Mood and driving: Is there an effect or affect? In T. Rothengatter and E. Carbonell Vaya (eds.) Traffic and Transport Psychology: Theory and Application. Pergamon, Amsterdam.

[3] Jamson, S., Wardman, M., Batley, R., \& Carsten, O. (2008). Developing a driving Safety Index using a Delphi stated preference experiment. Accident Analysis and Prevention, 40, 435-442.

[4] Lang, P.J., Bradley, M.M., \& Cuthbert, B.N. (2008). International affective picture system (IAPS): Affective ratings of pictures and instruction manual. Technical Report A-8. University of Florida, Gainesville, FL.

[5] Lonczak, H. S., Neighbors, C., \& Donovan, D.M. (2007). Predicting risky and angry driving as a function of gender. Accident Analysis and Prevention, 39, 536-545.

[6] Matthews, G. (2002). Towards a transactional ergonomics for driver stress and fatigue. Theoretical Issues in Ergonomics Science, 3 (2), 195-211.

[7] Matthews, G., Desmond, P.A., Joyner, L., Carcary, B., \& Gilliland, K. (1996). Validation of the driver stress inventory and driver coping questionnaire. Paper presented at the International Conference on Traffic and Transportation Psychology, Valencia, Spain, May 1996.

[8] Mesken, J., Hagenzieker, M.P., Rothengatter, T., \& de Waard, D. (2007). Frequency, determinants, and consequences of different drivers' emotions: An on-the-road study using selfreports, (observed) behavior, and physiology. Transportation Research Part F: Traffic Psychology and Behaviour, 10, 458475.

[9] Pêcher, C., Lemercier, C., \& Cellier, J-M. (2009). Emotions drive attention: Effects on driver's behavior. Safety Science, 47, 1254-1259.

[10] Stephens, A.N. \& Groeger, J.A. (2006). Do emotional appraisals of traffic situations influence driver behaviour? Behavioural Research in Road Safety XVI, 49-62.

[11] Stephens, A.N. \& Groeger, J.A. (2009). Situational specificity of trait influences on drivers' evaluations and driving behaviour. Transportation Research Part F: Traffic Psychology and Behaviour, 12, 29-39. 